\section{OBSERVATIONS ON THE ETIOLOGY AND MORBID ANATOMY OF TUBERCULOUS MENINGITIS. 1}

By EDMUND CAUTLEY, M.D. CantaB., \&C., PHYSICLAN TO THE BELGRATE HOSPTTAL FOR CHILDRN ; ASSISTANT PHYSICIAN TO THE METROPOLITAN HOSPITAL.

For the data of this paper I have taken the records of the last 27 cases of tuberculous meningitis which have been under my care during life and have been subsequently examined in the dead house (vide Table). Though the number is too small to justify dogmatic statements on any one point, the results obtained from the inquiry are sufficiently definite to enable me to bring before you certain deductions as to the etiology of the disease.

1. The age distribution. - The following table shows the age distribution :-

Age in years.

Males. 'Females.

Total.

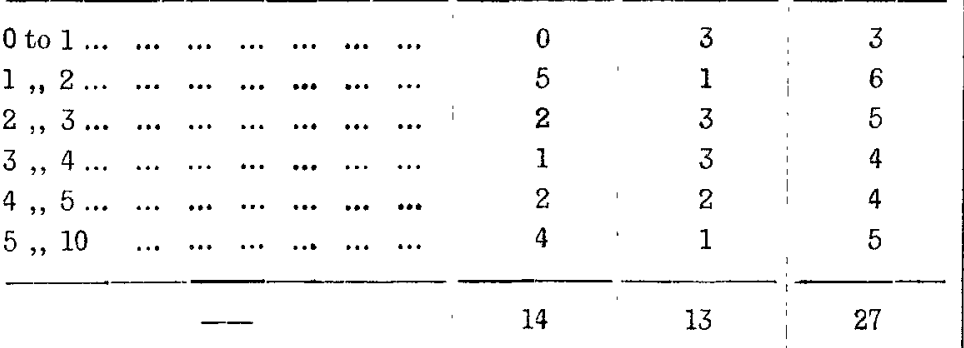

No less than 22 out of the 27 cases occurred during the first five years of life and only five during the second five years. This result confirms those of other observers that the disease is most common under five years of age. Some writers state that it is rare during the first year and give statistics in support of their statements. Others find it frequent during the first year. It is noi tlways clear, from the figures quoted, whether the statenuents are based on cases diagnosed during life or only on those which have been verified after death. If the figures are based on clinical records only it is probable that the frequency of the disease during the first year would be over-estimated, on account of the difficulty in invariably making an accurate diagnosis between the simple posterior basal meningitis, which is unduly prevalent during the first year of life, and true tuberculous meningitis. My own figures show that the distribution of the cases during these first five years is fairly equal for the different years. There is no preponderance of either sex-14 males to 13 females.

2. The influenoe of heredity. - In recent years it has become customary to ascribe very little importance to heredity in the production of tuberculous affections, except in so far as the sufferers are exposed to direct infection from association with a tuberculous relative, or may inherit a peculiarity of constitution which renders them susceptible to such infection. It is well known how difficult it is in hospital practice to obtain accurate family histories and that all statements as to the occurrence of "consumption" in the parents or other relatives must be viewed with suspicion. "The results obtained on this point are opposed to the theory of heredity but must not be too strongly insisted upon. In 22 of the cases it was definitely stated in the notes that there was no history of phthisis in the parents or other children. Of the remaining five cases a few facts may be given. In only one case was the mother stated to be affected.

CASE 14. - The patient was a female, aged 6 months. The mother was said to be very ill with "consumption and bringing up blood." The child was only nursed for one week and was then brought up on condensed milk. She had been taken out of doors very little. She died with sym ptoms of tuberculous meningitis, and there were found, in addition, tuberculous consolidation of the upper lobe of the left lung, a cavity in the upper lobe of the right lung, general dissemination throughout the lungs, and a few tubercles in

1 A paper read before the Society for the Study of Disease in Children on Nov. 15th, 1901 the spleen. No note was made as to the condition of the mediastinal or mesenteric glands.

No better instance could be given of probably direct infection from the mother through the respiratory tract The next four cases also illustrate the possibility of direct infection from a parent.

CAsE 2. - The patient was a female, three years and nine months old, who died after an illness of 19 days' duration. Tubercles were found in the meninges, liver, and spleen. No caseous glands were found. The cribriform plate of the ethmoid was carious. While the child was ill the father was an in-patient at the Brompton Hospital for Consumption.

The case suggests strongly that infection took place through the nose. To quote Usler: "In those instances in which no primary focus has been discovered it has been suggested that the bacilli reach the meninges through the cribriform plate of the ethmoid from the upper part of the nostrils, but this is not probable." It is certainly not probable that the meninges can be infected by this route unless disease of the bone is first set up. The possibility of direct infection is present in the next case, which also presents another point of interest, to be subsequently dis.cussed-namely, the influence of injury in the production of the attack.

CAsE 1.--The patient was a female, aged two years anc: six months, who died after an illness of 17 days duration. After death there were found basal meningitis, with only a few tubercles in the meninges, and no evidence of a tuberculous focus. A few pleural adhesions were present at the apex of the right lung. The child had a fall three weeks before the onset of the symptoms. The father was said to be "consumptive" and a sister was at the time in the Brompton Hospital for the same disease. It is a question whether the tubercles in the meninges were the primary cause or secondary to a basal meningitis set up by the fall.

The other two cases presented interesting features. In both the father was said to be "consumptive."

CASE 19. -The patient was a male, aged two years, who died after an illness of 18 days' duration. A large cavity was found in the lower lobe of the left lung; there were general dissemination throughout the lungs and caseous mediastinal glands.

CASE 24. - The patient was a male, aged three years and seven months, who developed left hemiplegia during the course of the illness. An old tuberculous mass was found in the right external capsule and tuberculous nodules in the cerebellum. The lungs were stuffed with tubercles and the mediastinal glands were caseous. Only one tubercle was present in the spleen.

These five cases were the only ones in which a family history of tuberculous disease was obtained and they all illustrate the probability of direct infection.

3. Injury as an etiological factor.-In only one instance (Case 1), that already referred to, could injury be invoked as the exciting cause of the disease. Even in this case it is possible that the meninges had become infected before the injury, for the presence of pleuritic adhesions at the right apex suggests former tuberculous mischief. All text-books recognise the importance of this point. It is more specially applicable in the case of children who go to school. For some reason or other the child receives a box on the ear, or a rap on the head by the knuckles or ruler or other punitive instrument in the hands of the teacher. Subsequently the child dies from tuberculous meningitis which is ascribed by the parents to the injury. Possibly the unfortunate teacher is hauled before a coroner's court and severely censured by a typical British jury. The common and usually accepted explanation is that the child was punished for the stupidity or inattention, the state of mind so likely to be present during the early stages of the disease. It is therefore imperative in such cases that a careful post-mortem examination should be made in order to ascertain whether there is a tuberculous focus present which might have given rise to the meningeal infection. On the other hand, a case like the one quoted might be brought forward as an argument in favoul of injury being the exciting cause. Injury to the brain or meninges might cause local congestion or damage of such a nature as to render the affected part a suitable soil for the growth of the tubercle bacillus, which without such advantageous conditions might have perished. This is the only instance out of the 27 cases which is favourable to this view.

4. The ohannel of entrance of the broillus into the system.In view of the recent discussions and observations on the 
Table giving Details of Necropsies in 27 Cases of Tuberculous Meningitis.

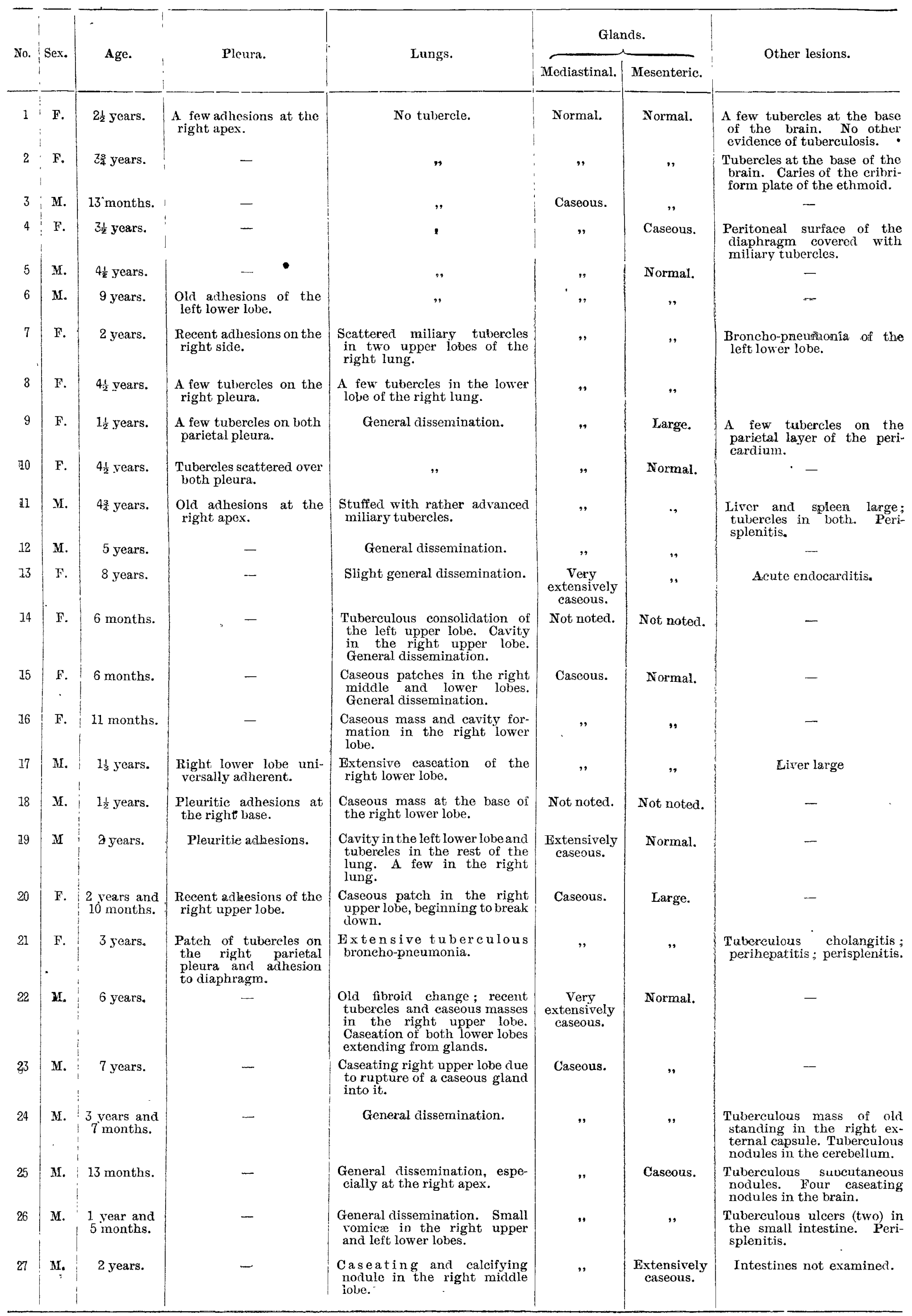


1726 The Lanoet,] DR. F. CAUTLEY: ETIOLOGY, ETC., OF TUBERCULOUS MeNiNGiTiS. [Dec. $21,1901$.

relationship of the bacillus of bovine tuberculosis to that of the human disease, and on the infectivity of the milk of tuberculous cows, this point is one of extreme importance. In the last edition of Fagge's Text-book of Medicine (vol. i., 1901), edited by Pye-Smith, it is stated in the chapter on Tuberculous Meningitis that "the exciting cause is the entrance of Koch's bacillus into the circulation, and in children this is probably by way of the intestinal tract from the milk of tuberculous cows." Kanthack, Delépine, and others have proved that the bovine tubercle bacillus is frequently present in cow's milk. It has been found by Bang, Bollinger, and others in the milk of cows affected with general tuberculosis or tuberculous disease of the udder. It has been experimentally determined by Hirschberger that the milk of tuberculous cows may be infective, even though the udder is unaffected. Kempner and Rabinowitsch in 1899 found the bacillus in the milk of cows which reacted to tuberculin but gave no other evidence of tuherculosis, and their results have been confirmed by Ostertag, Adami, and Martin. There is, in fact, no shadow of doubt that the bovine tubercle bacillus is often present in cow's milk. One warning must be given on this point. Acid-resisting bacilli similar in nature and appearance to the tubercle bacillus have been also found in milk (Petri, Beck, Santoni, Rabinowitsch, \&c.), in the udder (Ludwig Neufeld), in the intestines of healthy and tuberculons cows (Olt), in the timothy grass sometimes used for fodder (Moëller), and in the dung (Severin). These bacilli cannot be distinguished microscopically but can be readily distinguished by intra-peritoneal injection into guinea-pigs. Tuberculosis can he produced in calves and pigs by feeding them on tuberculous milk, and by analogy it has been assumed that a similar result will arise in children. In support of this the reports of the Registrar-General are often quoted. In these reports it appears that the disease called "tabes mesenterica," usually regarded as a tuberculous affection, is increasingly prevalent among infants. With regard to this latter statement I wish to enter a very strong protest. 'labes mesenterica is very rare among infants, and there is little doubt that almost all the cases so entered in mortality statistics as occurring in infants under one year of age are neither more nor less than the interesting disease known to the public by that blessed name "consumptive bowels"that is, diarrhoea and wasting due to improper feeding and ending in death. I have never yet seen a true case of tabes mesenterica in an infant examined post mortem, although I have been constantly on the watch for one, and I am strongly convinced that it would conduce to scientific accuracy if the name were eliminated from our nosology.

To return to the point under consideration. The main channels of infection are the respiratory and the alimentary tracts. Of quite secondary importance are the ear and skin, both of which occasionally prove channels of infection. Let us consider, then, what evidence these post-mortem records yield in favour of one or other of these modes of entry of the bacillus. Using the term "mediastinal glands" to include all the glands in the anterior and posterior mediastinum, at the bifurcation of the trachea, and at the roots of the lungs, a most striking fact is at once revealed. In only two instances were these glands normal and in these, the tuberculous process was limited to the base of the brain, being in one of them associated with caries of the cribriform plate of the ethmoid, and in the other possibly secondary to a localised pleurisy at the apex. In two other instances (Cases 14 and 18) no note was recorded as to the condition of these glands, but $I$ have little doubt that, considering the advanced tuberculous disease in the lungs, the glands must almost certainly have been affected. In the remaining 23 cases one or more of the mediastinal glands was caseous. The one most often affected, frequently the only one, was the gland situated at the bifurcation of the trachea, commonly called the pretracheal gland. In four of these 23 cases the mesenteric glands were also caseous. In one of these four (Case 4) the peritoneal surface of the diaphragm was covered with miliary tubercles and there were tubercles in the spleen, while the lungs only showed evidence of bronchitis. The other three cases, in which both sets of glanás were caseous, are of much interest.

CASE 25.-The patient was a male, 13 months old, who was under my care for two months for subcutaneous tuberculous nodules. There were four such nodules, varying in size from a split pea to a horse-bean, one of which was said to have been in existence since the age of four or fire months. He developed meningitis from which he died. In addition to tuberculous basal meningitis the brain was found to contain caseating nodules, the lungs showed general dissemination, especially at the right apex, and there was extensive caseation of the mediastinal and mesenteric glands, with a few tubercles in the spleen.

In the other two (Cases 26 and 27) there was evidence to warrant the assumption that the abdominal tuberculosis was secondary to the swallowing of tuberculous material coughed up from the lungs. Thus, in a boy, aged 17 months. tuberculous vomica were found in both lungs and two small tuberculous ulcers in the small intestines. In a boy, aged two years, an old caseating and calcifying nodule was found in the lung, but unfortunately the intestines were not examined. In nine instances there was more or less dissemination in the lungs, or in the lungs and pleura, but no evidence of old-standing clisease. In only one of the nine, the child with subcutaneous nodnles, were the mesenteric glands caseous. In 12 instances the lungs showed extensive tuberculous disease of old standing-namely, easeation and in some cases excavation.

Looking at the facts from another point of view it seems somewhat remarkable that, if the alimentary canal is so liable to tuberculous infection as the upholders of the theory of infection by tuberculous milk would have us believe, in only two out of these 12 cases in which the disease was advanced and of old standing in the lungs was there evidence of infection of the mesenteric glands. In two the state of these glands was not noted. Of course, it is clear that such infection may have been present and that microscopical or experimental evidence might have proved some of these glands infected; but even if this were so, the facts strongly confirm the view that the respiratory tract was the primary source of infection. It is also clear that the alimentary tract is not markedly susceptible to infection by the human tubercle bacillus.

It has been stated that the tubercle bacillus of cattle is not dangerous to human beings. Even if we only accept the statement in a modified form and say that the bovine bacillus is not nearly as infective as the human bacillus, our postmortem facts afford a still stronger proof of the insusceptibility of the alimentary tract to infection by the bovine bacillus, seeing that it so often escapes although exposed to the risk of infection by the human bacillus in chronic tuberculous disease of the lungs. In young children, as is well known, sputum is swallowed and not expectorated. Consequently the liability to secondary infection of the alimentary canal is enormous, whereas the post-mortem evidence proves it to be infrequent. In six instances was there no evidence of tuberculous disease of the lungs and yet in four of these the mediastinal glands were caseous. In one of the four the mesenteric glands were also caseous. It is possible that in these cases a small tuberculoús lesion may have healed and left no trace visible to the naked eye or that the bacillus reached the gland through some other channel. In some instances in which the lung was affected the question arose whether the lung mischief was primary or secondary to an extension from the glands. In one case it was clear that the main part of the lung disease was due to the actual rupture of a breaking-down gland into its substance (Case 23). In some others the tuberculous process could be seen extending backwards from the glands along the lymph channels in a radiating fashion into the lungs. The probable sequence of events in most cases is that the bacillus is oarried by the blood or lymph stream to a congested gland and there develops, finally causing caseation. Such a caseous gland may remain quiescent, may become calcified, may break down, may ulcerate through into the trachea or cesophagus, or may rupture into the lung or pleural cavity. In any case if it breaks down the infective material may become widely disseminated and cause a general infection. The post-mortem evidence may be summed up shortly as proving that the respiratory tract is the great channel of infection. It therefore, follows that the danger from swallowing tuberculous cows' milk has been much exaggerated.

It is sometimes asserted that the bacillus can get into the system through the alimentary canal without producing a local lesion. Caseous mesenteric glands are found although the mucous membrane of the intestines appears normal. If the bacillus does not pass out of the intestines through the lymph channels to the glands it might possibly pass into the blood stream. Practically all the blood from the intestines goes through the liver. We should expect, then, that if the bacillus passed into the blood stream it 
wonld produce tuberculous disease of the liver. Miliary tubercles are indeed found in the liver, more often in the spleen, and less often in the kidneys, but only when there is considerable general dissemination throughout the body. It is rare to find old tuberculous foci in the liver and in none of these cases was such a focus found. There is no evidence at all strongly in favour of infection of the blood stream through the intestinal tract.

5. Evidence derived from the dist of the patients.-In eight out of 13 infants under three years of age the child was brought up on the breast for the greater part or all of the first year of life. In four the mode of feeding was not stated. In one the child was brought up on condensed milk, for the mother was too ill from tuberculous disease of the lungs to nurse it. These facts, as far as they go, are suggestive that cow's milk is not a very virulent source of infection, for a very large number of infants are brought up on cow's milk. During the last 10 years I have brought up a large number of infants on cow's milk, and as far as I know not one of them has subsequently come under my care for tuberculous meningitis. The milk has been boiled if the mothers have carried out the directions given.

6. The state of the brain as indicative of the prospect of perative treatment proving beneficial.-In about one-third of the cases there was no excess of fluid in the ventricles. In the remainder the fluid varied from a slight excess to a large amount. In many instances the cerebral tissues were very soft and occasionally almost diffuent. Operative treatment can only be indicated as a means of relieving pressure and under the assumption that the child is dying from the coma induced by pressure. Such is not always the case, for many children died comatose in whom there was no evidence of increased intracranial pressure after death. The coma is probably due to the softening of the brain which may be associated with excess of fluid in the ventricles or may occur quite independently. Hence it is clear on anatomical grounds that operative treatment would only relieve pressure in about one-third of the cases, and that even in these cases it would do little or no good on account of the softening so often present and which is dependent upon insufficient bloodsupply rather than on an excess of fluid. Moreover, these records indicate how very rarely the disease is limited to the brain and that the operation would only afford temporary alleviation of symptoms.

7. Classification. - It is possible to divide the cases on anatomical grounds into three gromps: (1) simple tuberculous meningitis, in which the disease is limited to the brain (very rare); (2) tuberculous meningitis, secondary to a localised tuberculosis with little dissemination (the child dies from the meningitis) ; and (3) general miliary tuberculosiscases in which the meningeal symptoms bear a comparatively unimportant relationship to the disease.

To conclude, I am strongly convinced that inheritance means exposure to infection of a weakly or predisposed child; that injury is very rarely an exciting or predisposing cause; that the respiratory tract is the great channel of infection; that the alimentary tract is rarely primarily infected ; that cow's milk is very rarely, if ever, the source of infection; that limitation of the tuberculous process to the meninges is very rare ; that the prognosis is very hopeless on account of the extent of the tuberculous disease elsewhere ; and that operative treatment may be discarded as experimental rather than useful.

Upper Brook-street, W.

\section{OVARIOTOMY IN PREGNANCY : THREE RECENT CASES.}

BY JOHN B. HELLIER, M.D. LOND.,

HONURARY OBSTETRIC PHXSICTAY TO THE LEEDS GENERAL INFIRMARY AND LECLURER ON DISEASES OF WOMEX AND CHILDREN IN THE YORKSHIRE COLLEGE, VICTORLA VNIYERSTTY.

CASE 1. Ovariotomy at the third month of pregnancy; full term dclivery. - A married woman, aged 32 years, was admitted into the Leeds General Infirmary on Nov. 29th, 1900 , for ovarian tumour. She complained that the abdomen had remained large since her last confinement, two years previously, and had steadily increased in size. She had missed two periods. The abdomen was distended by a large ovarian cyst reaching to within three fingers' breadth of the sternum; the umbilical girth was $35 \frac{1}{2}$ inches. Fluctuation and thrill were very perceptible. On vaginal examination the uterus was felt lying anteverted, with a bulky fundus felt through the anterior vaginal wall. The cervix was soft; there were vulval and mammary signs of pregnancy. Ovariotomy was performed on Dec. 1st. The cyst, which contained 164 ounces, was large, multilocular, and non-adherent the pedicle was from the left side. The right ovary, which was enlarged but not cystic, was not removed. The uterus was about three months pregnant and felt like a flaccid cyst. The wound healed by first intention and the patient made a good recovery with no threatening of abortion. She went to full term and bore a living child.

CASE 2. Ovariotomy at the fourth month of pregnancy; full-term delivery. - A married woman, aged 34 years, was sent to me by Mr. W. F. Chrispin of Castleford. Her last confinement was in July, 1900 ; menstruation returned in September and ceased again at Christmas. She had noticed an increase in the size of the abdomen about Jan. 1st, 1901. She was admitted into the Leeds General Infirmary on March 4th. She said that she had been married 15 years, had had 13 pregnancies and no miscarriages, but only one of her children had lived for more than 13 months. Her own health and general condition were good. The abdomen contained a uterus four months pregnant and a mobile tumour in the umbilical region which seemed to be loosely connected with the uterus. The tumour was rather solid on palpation. The usual signs of pregnancy were present. Ovariotomy was performed on March 9th and the tumour proved to be a semi-solid multilocular cystorna with the pedicle from the left of the uterus. There was extensive adhesion to the omentum and small bowel. The right ovary was normal. The uterus was four months pregnant. The operation was completed in the usual way; no drainage was employed. A continuous catgut suture was used for the peritoneum. The patient made a good recovery, except that there was some suppuration in the wound and the catgut came away entire and unabsorbed 11 days after. She had also a roseolous rash on the fifth and sixth day, which was followed by some desquamation. There was no threatening of abortion. She went to full term and bore a living child on Sept. 1st and made a good recovery.

CASE 3. Ovariotomy for dermoid cyst in the sixth month of pregnanoy; still-born child at the eighth month.-This is a very interesting case. On May 13th, 1901, Mr. R. Smailes of Leeds asked me to see with him a patient, aged 42 years, who was five months pregnant and in whom he had discovered a post-uterine tumour. She had had three previous normal pregnancies and two miscarriages, the last being in the summer of 1899. After this she menstruated regularly till December, 1900, when she became pregnant. When two months pregnant she had some vaginal coloured discharge and the uterus was found to be retroflexed, and a pessary was applied. In April an examination was made to determine the position of the uterus, and the tumour was found in the pouch of Douglas. When I saw the patient she was a healthy woman in good condition and had no symptoms to suggest that pregnancy was abnormal. On vaginal examination an elastic cystic mobile tumour was found in the pouch of Douglas. It could be pushed up but soon returned. It seemed to be almost certainly ovarian and its removal was strongly advised, as it might cause serious complication in labour if it was left alone. Ovariotomy was performed in a nursing home on May 27th, Mr. Smailes administering chloroform, and Mr. C. H. Moorhouse assisting me. A median incision exposed the large flaccid uterus reaching to the umbilicus. The pedicle sprang from the right side; the oviduct was so greatly enlarged that it had to be examined carefully to make sure that it was not small intestine which it resembled. As traction on the pedicle did not easily withdraw the cyst the hand was introduced around the flaccid uterus and the cyst was extracted entire. The pedicle was twisted, the tumour was deeply congested, and there was free ascitic fluid. The left ovary was normal. The operation was concluded in the usual manner, with a continuous catgut suture through the peritoneum and interrupted silkworm gut through the skin and muscle. The tumour was a dermoid cyst containing hair and sebaceous material and of about the size of an orange. During the first 24 hours after the operation the patient had more severe abdominal pain than I ever had observed after a successful abdominal section. She described the pain as agonising. There were no other bad symptoms and morphia administered hypodermically relieved the pain. She made an excellent recovery. The wound healed by first intention and she went home on an 